\title{
The effect of atmospheric plasma jet on Fusarium species producing mycotoxins T2 and DON: An approach for physical and chemical investigation
}

\author{
Elham Galin Abbasianª, Mansour Bayata,*, Arash Chaichi Nosratib, Seyed \\ Jamal Hashemic, Mahmood Ghoranneviss ${ }^{\mathrm{d}}$ \\ a Department of Microbiology, Science and Research Branch, Islamic Azad University, Tehran, Iran \\ ${ }^{b}$ Division Microbiology, Department of Molecular \& Cell Biology, Faculty of Basic Sciences, Lahijan \\ branch, Islamic Azad university, Lahijan, Gilan, Iran \\ cTehran University of Medical Sciences, Tehran, Iran \\ dPlasma Physics Research Center, Scince and Research branch, Islamic Azad University, Tehran, \\ Iran
}

Received: 23 September 2019, Accepted: 15 November 2019, Published: 02 December 2019

\begin{abstract}
Fusarium genus is a filamentous ascomycete with the ability to produce toxins. Sampling was carried out between 2015-2016 in three tea courses from Mazandaran and Gilan provinces. At intervals of 3, 7 and 15 days, the plates were examined and the colonies grown in each plate were identified and their macroscopic characteristics were recorded. Finally, 150 colonies were selected. Samples cultured in a plate were cultured in liquid medium $\mathrm{sB}+\mathrm{ME}$ and $\mathrm{sB}+\mathrm{yE}$. The environments were examined for successive days and slides were prepared and stained with lacto phenol. This is to ensure the inactivation of fungi exposed to plasma or the removal of spores from the sample surface. Results showed a significant relationship between the DON produced in the potato medium containing yeast extract and DON contained in the sample taken from the same medium that was exposed to plasma jet for 60 seconds. This feature was statistically significant in comparison between changes in the toxicity of $\mathrm{T} 2$ and toxin after 60 seconds of treatment with jet plasma and T2 reducibility severity to DON. Plasma treatment showed a significant reduction in time-dependent toxin concentrations. These results indicated that the plasma system has a high potential not only to destroy mycotoxins, but also toxins produced from fungi, which can be greatly reduced and can be used effectively in the food industry.
\end{abstract}

Keywords: Fusarium; DON; T2; mycotoxins; plasma jet.

\section{Introduction}

The term "fungi" refers to all factors that are classified in the fungi, although fungi and algae were formerly introduced as early crops, but are now characterized by increasing information on creeping cycle, physiology, genetics and biochemistry which cannot be classified

*Corresponding author: Mansour Bayat 
in the flora and fauna. Fungi are nonmoving organisms with nuclei and cell walls, lacking chlorophyll pigmentation and replicating by spores. Spores which are produced sexually and non-sexually are small (microscopic) and lacking embryos. Fungal spores, if they are in a favorable environment and conditions, grow and multiply produce molds $[1,2]$. Hence, fusariosis, caused by species, is an important fungal disease that is persistent in its treatment and is often caused by Fusarium solani. Fusarium, respiratory and skin species are introduced into the species. The main source of infection are skin, blood and lungs, respectively. The fungus engulfs the organs of the body such as the lungs, the heart, the liver, the spleen, and the kidneys after entering and deploying through the blood in the disseminated body. Fusarium threatens the life of patients with leukemic disease or aplastic anemia [3]. Studies in Iran and elsewhere indicate the high prevalence of Fusarium fungus and especially Fusarium verticillioids. Khosravi et al., isolated Fusarium mushrooms in six percent of samples from maize, barley, and maize silage. This mushroom is a famous mushroom farm. In terms of the amount of produced toxin produced by this group, the toxins produced by this fungus are called mycotoxins. Mycotoxin is derived from the Greek word myke, meaning mushroom and toxicum, meaning poison. Mycotoxins are a mixture of toxic compounds produced by fungi. Mycotoxins, along with other fungal metabolites, such as antibiotics, alkaloids, and the like, are compounds that produce fungal fungi in the late stages of fungal growth. Such metabolites, known as secondary metabolites, seem to have no benefit to the fungal cells themselves, and they are used in principle as a defense mechanism.
Dioxynvalenol (DON) is one of the several mycotoxins produced by Fusarium species that causes contamination of corn, wheat, barley, rice and other cereals during storage of these substances and is a risk factor for humans and animals. This toxin has been known as a nauseating drug that causes vomiting, and was first reported in Japan in 1972 after mink juice consumption. Toxin T2 is also a form of diabetes mellitus of HT-2 toxin and a member of the family of trichothecans, a common tetracyclic loop produced by Fusarium species, invading grains and inhibiting protein synthesis and cell proliferation in plants. In humans and animals, they also cause metabolic changes. T2 toxin is one of the most extensive combinations of the Fusarium poia family and the sporozoic follicular furosemitis genotypes, and is a major contribute to the development of food allergy to humans, all of which are related to the development of membrane systems. If this stage is achieved, patients receive a healthy diet without being contaminated with vitamins. However, the continued exposure to toxin causes damage to the bone marrow and the hematopoiesis, leading to anemia and a decrease in the number of erythrocytes and platelets [4]. Toxin T2 is highly poisonous. The amount of this poison in corn silage is higher than other components of the feed. Toxin T2 is also thought to be one of the toxins involved in human disease called ATA and is said to be one of the compounds of yellow rain collected in Southeast Asia. Therefore, we study the effect of atmospheric plasma jet on the reduction of mycotoxin produced in this study. The effect of the plasma can be completely selective, that is, between the damage to the pathogen microorganisms and the pathogen without damage to the activation of different pathways in different microorganisms [5-7]. Low 
oxygen pressure plasma decomposes lipids, proteins, and DNA of the cells.

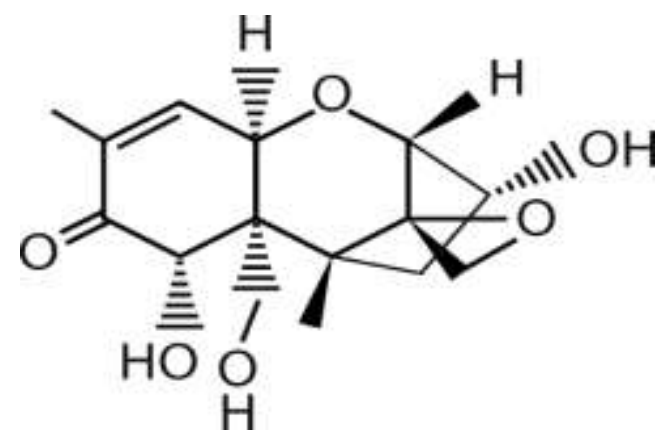

Figure 1. DON chemical structure

Plasma reactive specimens are widely associated with direct oxidative effects on the outer surface of the microbial cells. For example, as a rule, the consumption of oxygen and nitrogen in plasma is a great source of nitrogen and reactive oxygen base samples such as $\mathrm{O}$, $\mathrm{O} 2, \mathrm{O} 3, \mathrm{OH}, \mathrm{NO}, \mathrm{NO} 2$, and so on. Microorganisms are exposed to radicals in the plasma method for intense bombardment to cause severe surface damage, so that the living creature cannot be recovered sufficiently and quickly. Concerning the same use of the production or manufacture of mycotoxins; numerous false reports have been made on the production of aflatoxins. For the first time, Kung et al. (2009) stated that cold plasma is a very effective killer against bacteria. Also, cold atmospheric plasmas have the ability to disable general microorganisms under controlled laboratory conditions [8]. Yang et al. stated that plasma causes protein degradation and hyper bili rubinization of the gram-negative bacteria of Escherichia coli, resulting in the destruction of microorganisms by bacterial membrane degradation [9].

Some of the chemicals capable of destroying aflatoxin B1 include chlorinecontaining agents such as sodium hypochlorite and oxidizing agents such as hydrogen peroxide, ozone, sodium bisulfite and sodium sulfide. These chemicals oxidize the double bond of the eruption end ring or hydrolysis and oxide the lactone ring. The general purpose of plasma is to produce energetic or quasistable particles in the gas, such as the excited states of atoms, ions, and molecules that are chemically active. The presence of such energetic, metastable particle species causes various surface phenomena such as (reaction engraving) or saturation, which does not occur without the presence of plasma and only by heat [10]. In most plasma processes, they return to their species immediately because of the low ionization fraction of atoms and ions that they have lost. The aim of the present study was to investigate the effect of atmospheric plasma jet on Fusarium species producing mycotoxins $\mathrm{T} 2$ and DON.

\section{Materials and methods \\ Sampling}

Samples were taken in May of 1394 to the end of October 2016. This sampling was carried out in three periods of tea cultivation from Gilan and Mazandaran provinces using open and closed positions. In general, 6 plates containing Extract Agar Malt, Extract Agar, Extract Oxygen Extractor, Phoxic Agar, Sabo Dextrose Agar, and Putito Dextrose Agar were all mixed with chloramphenicol $100 \mathrm{ppm}$ and tetracycline $50 \mathrm{ppm}$, which prevailed over three to five days in 25 Centigrade degrees used for samples. Plates containing $25-15 \mathrm{~cm}$ cubic meters of agar (12-10 cm in diameter) after 30 , 60 and 90 minutes of field (451 remaining plots in the fields) and 15,30 and 60 minutes from factories (441 plates Remaining in factories), nearly 900 plates were removed and then prepared in perforated polyethylene bags for transfer to a lab. All plates were incubated at $25 \pm 2{ }^{\circ} \mathrm{C}$ and aerobic conditions. In this way, a plate of each package was stored in dark conditions, 
one in light conditions and one pair of plates in the dark. Two remaining residue plates were stored in each refrigerator (8-4 centigrade) for each replacement. At intervals of 3, 7 and 15 days, all plates were examined and the colonies grown in each plate were identified and labeled with a stereoscope and isolated using a sterile glass needle and cultured in preprepared plates. Then, their microscopic and macroscopic characteristics were recorded, which eventually resulted in 150 colonies.

\section{Cultivation and isolation}

At first, 3 Sabo dextrose broth $(25 \mathrm{cc}$ ) + Extratat malt $(7.5 \mathrm{~cm})$ and Sabo dextrose broth $(25 \mathrm{~cm})+$ extrathect $(7.5$ $\mathrm{cm}$ ) and Phoxic Agar (Escherichiosus environments) were cultured in accordance with the instruction on the cans of the cultivated environments. the photocopier was divided in the plates, and the liquid environments were placed in the falcon and prepared after the preparation of the culture media mentioned above and then the specimens were prepared in a photochemical agar medium as needle cultivation (At 3 points, $2 \mathrm{~cm}$ apart and at the edge of the plate). Next, we incubated at $25^{\circ} \mathrm{C}$. Finally, on the fifth day after cultivating the slides, we examined the cultures and cultivated plates so that the sliders of the calcine should not be dried and if distilled water was dried, we added distilled water again. After control, on consecutive days, on the tenth day after reintroduction, media were checked for solid, solid and slide calcinations. Then, on the $14^{\text {th }}$ day, the Falcon tubes containing the fluid culture medium were vortexed once for 15 minutes and then vortexed at $70{ }^{\circ} \mathrm{C}$ once after one time. Then, after freezing inside each falcon, a 2.5 milliliter extraction solution $(100 \mathrm{~mL}$ of acetonitrile +20
$\mathrm{mL}$ of acetone $+100 \mathrm{~mL}$ of ethanol + $100 \mathrm{~mL}$ of methanol) was added and then the vertex phase was repeated again and this was done for 15 minutes, then again, we added 2.5 extraction solutions; afterwards, we repeated the vertex for 15 minutes. On the fourteenth day, the slurry slides were prepared on a cassette so that on each slide, where the two parts of the slurry were repeatedly cultured, the culture medium was discarded and the lactobhenol paint was poured over both portions (one drop) and sterilized lamellum. We placed the lamellas on each part of the collet on a sterile slab that was lactobencholic in two portions (1 drop), (infected samples on those parts). The next day, they were examined by X-ray, X-40, and X-100, and photographed in an optical laboratory. Then, after the diagnosis, we prepared solutions that were extracted from the solid and liquid medium for the ELISA method. Then, they were placed in Ben-Mari at $90{ }^{\circ} \mathrm{C}$ for 10 minutes to remove the extraction solution and then the specimens were examined.

\section{ELISA procedure}

We performed the ELISA method according to the brochure kit, and then, in the next step, we placed the samples in a cold plasma jet at concentrations determined from plasma jet at 30, 60 and 360 seconds. At this stage, the sample volume of the solution decreases, which is reduced to volume conversion to a specific proportion of distilled water, and then returned to the microtubules, which is determined by the ELISA method to determine the effect of the plasma jet on the sample.

\section{Cleansing mycotoxins with atmospheric cool plasma jet}

In this study, a device for purifying mycotoxins was used that produced 
plasma jet which had the greatest impact on mycotoxins. In this research, plasma jet system of Islamic Azad University, Science and Research Branch of Tehran was used. In this device, the chamber in which the plasma is formed is made up of a helix to make the plasma jet most in contact with the surface of the mycotoxin and to act as a plasma reactor. The length of the glass tube used in the production of Helix is $12 \mathrm{~cm}$ and its internal diameter is $2 \mathrm{~mm}$ and its outer diameter is $3 \mathrm{~mm}$. A glass spiral was made with a core diameter of $3 \mathrm{~cm}$ and a step spacing of $1.5 \mathrm{~cm}$. During the experiments, argon gas was chosen as the main gas used to make the plasma. First, the output of the power supply was adjusted to $50 \mathrm{~kW}, 100 \mathrm{~W}$, and the electron frequency of $30 \mathrm{kHz}$ (optimal conditions for stable and effective plasma) and their exact values were measured with high voltage probes and oscilloscopes. Then, with the gas flow (gas speed of 6 liters per minute) and the high potential difference between the two connected electrodes, the effect of cold atmospheric plasma (argon-air) was investigated for 30, 60 and 360 seconds. The experiments were performed in three replications.

\section{Statistical analysis}

Analysis of Wilcoxon correlations and frequency of variance for determining significant differences between the mean of tests in $\mathrm{p}=5 \%$ were used to perform multiple comparisons based on the least significant difference (LSD). A value of $\mathrm{P}<0.05$ was considered to be statistically significant.

\section{Results and discussion}

In this study, we investigated the derivative effects of Aragon plasmainduced micro-waves against fusarium mycotoxins such as DON and T2 toxins. Plasma treatment results showed a significant decrease in the concentration of time-dependent toxin. The successful use of DAPACP in sterilization methods depends on the amount of gas and flow used and the released radicals that naturally react with the sample. In addition, inhibition of Fusarium and its toxins produced, such as T-2 and DON toxins, depends on the accuracy of the device and on the distance where the sample is exposed to the plasma. The use of Aragon gas plasma in this study was successfully associated with the goal of inhibiting Fusarium and its toxins inhibition. Therefore, by advancing the use of cold plasma for food decontamination, it is possible to improve the effective and useful efficiency of this device in improving food quality in terms of contamination with microorganisms. Although the physicochemical properties and structural changes of plasma-treated mycotoxins were not defined, this study showed that the plasma system required certain conditions such as timing of effect and concentration of toxin in the nutrient to remove and inactivate the mycotoxins.

Considering that there was a significant difference between the numerical value and the mean size of $\mathrm{T} 2$ poison resulting from the growth of the fungus studied in the potato yeast extract medium in terms of the amount of the same poison produced in the environment, which was not statistically significant, A similar alignment that has been statistically significant has highlighted the fact that the amount of $\mathrm{T} 2$ contamination in the environment containing the extract of malt after 60 seconds of plasma treatment is greater than the toxic content produced in the yeast extract medium. The effect was accepted and reduced, which may be due to the tendency of the fungal samples studied 
to produce DON poison in Moore environments. It takes more use of $\mathrm{T} 2$ production in the same environments and in the same conditions, which requires further investigation.

Table.1. The results of DON concentrations obtained from analysis of LOG / LIN tables in P / M and P / Y media

\begin{tabular}{|c|c|c|c|c|c|c|}
\hline \multirow[b]{2}{*}{$\log / \mathbf{L I N}$} & \multicolumn{2}{|c|}{ Before Treatment } & \multicolumn{4}{|c|}{ After Treatment } \\
\hline & DON & DON & DON & DON & DON & DON \\
\hline Code & $\mathrm{P} / \mathrm{M}$ & $\mathrm{P} / \mathrm{Y}$ & P/M 30 & $\mathrm{P} / \mathrm{Y} 30$ & P/M 60 & $\mathrm{P} / \mathrm{Y} 60$ \\
\hline V1 & 0.22 & 0.22 & & & 0.596 & 0.931 \\
\hline $\mathrm{V} 2$ & 0.39 & 1.59 & & & 0.732 & 1.039 \\
\hline V3 & 1.23 & 0.99 & & & 0.749 & 0.997 \\
\hline V4 & 0.23 & 6 & & & 0.625 & 0.672 \\
\hline V5 & 0.49 & 0.22 & & & 0.935 & 0.918 \\
\hline mean & 0.512 & 1.81 & & & 0.727 & 0.911 \\
\hline
\end{tabular}

Table 2. Results of T2, DON concentration measurements obtained from analysis of LIN / LOG tables in $\mathrm{P} / \mathrm{M}$ and $\mathrm{P} / \mathrm{Y}$ environments

\begin{tabular}{ccccccccc}
\hline & \multicolumn{3}{c}{ Before treatment } & \multicolumn{4}{c}{ After Treatment } \\
\cline { 2 - 8 } lin/log & DON & DON & T2 & T2 & DON & DON & T2 & T2 \\
Code & P/M & P/Y & P/M & P/Y & P/M 60 & P/Y 60 & P/M 60 & P/Y 60 \\
V1 & 0.222 & 0.222 & 0.303 & 1.036 & 0.596 & 0.931 & 0.336 & 0.548 \\
V2 & 0.317 & 1.661 & 0.457 & 6 & 0.732 & 1.039 & 0.994 & 0.469 \\
V3 & 1.32 & 1.064 & 6 & 1.342 & 0.749 & 0.997 & 0.377 & 0.586 \\
V4 & 0.222 & 6 & 0.248 & 0.843 & 0.625 & 0.672 & 0.546 & 0.716 \\
V5 & 0.377 & 0.222 & 1.342 & 0.843 & 0.935 & 0.918 & 0.346 & 0.539 \\
mean & 0.492 & 1.833 & 1.67 & 2.012 & 0.727 & 0.911 & 0.402 & 0.572 \\
\hline
\end{tabular}

With more attention to the existence of a significant non-correlation between the concentration and mean DON of POTATO yeast extract in comparison to the DON produced in potato yeast extract, which was exposed to plasma for 60 seconds, the results are as follows: $(\mathrm{Sig}=0.045 / \mathrm{pc}=-$ $0.821)$. Thus, it is possible to reduce the amount of toxin by plasma irradiation, and on the other hand, the correlation between DON and $\mathrm{t} 2$ measured in samples previously influenced by plasma for 60 seconds which makes it clear that T2 toxin is more than DON under destructive action. Plasma jet will be placed in another study that should detail its further examination (Sig=0.013/pc=-0.906). The amount of DON toxin was produced in potato yeast extract compared with the T2 value of the same environment after a
60 second treatment correlated statistically. On the other hand, such a relationship was also statistically significant between the amount of DON poison dropped in comparison to the reduced $\mathrm{T} 2$ in the potato yeast extract after 60 seconds of plasma treatment, although it was not consistent $\quad(\mathrm{Sig}=0.013 / \mathrm{PC}=-0.906)$. There is a significant uncertainty and significant difference between the amount of DON poison in the T2 potato malt extract environment in the same environment $\quad(\mathrm{Sig}=0.028 / \mathrm{Z}=-2 / 201)$. This indicates that the fungal isolates studied during the incubation period have converted large quantities of their produced DON into T2. Comparing the reduction in the amount of $\mathrm{DON}$ in the potato malt extract medium compared to the potato yeast extract, there was a significant difference in the number of 
plasma treatments after 60 seconds with the incompatible and significant correlation, (Degree of numerical difference $=$ Sig=0.046, $Z=-1 / 992$ ). A significant difference was found between the numerical differences in the number of $\mathrm{T} 2$ toxin in the potato yeast extract medium with the same number of poisons in the same medium that was exposed to the plasma for 60 seconds $\quad(Z=-2 / 201$ ، $\quad$ Sig=0.028). Therefore, it can be shown that a sharp drop in $\mathrm{T} 2$ in a potato yeast extract after 60 seconds of treatment with a jet plasma has a significant discrepancy and is more intense than DON. There is a significant discrepancy between the amount of DON toxin and the decrease in T2 that occurs after 60 seconds of treatment with PlasmaGyte and is statistically significant. The findings are as trusted as possible $(Z=-1 / 992$, Sig=0.046).

\section{LOG/LIN wilcoxon test (Wilcoxon): V1 with V10}

The concentration of $\mathrm{T} 2$ produced in the potato malt extract with the concentration of DON produced in the potato malt extract is equal to the isolates; only the toxins differ. The difference between the mean of the numerical values is inconsistent and is statistically significant ( $\mathrm{Sig}=0.043$ ، $\mathrm{z}=-$ $2 / 023$ ) indicating that the amount of production and conversion of DON to T2 was statistically significant. The numerical difference between the reduced DON content in two potato malt extract media after 60 seconds of plasma treatment indicates that poison in the potato malt extract medium is reduced further, $(\mathrm{Sig}=0.028, \mathrm{Z}=-$ 2/201). This feature was statistically significant in comparison with the variation of toxic effect of $\mathrm{T} 2$ and toxin after 60 seconds of plasma jet treatment and T2 degradation severity compared to DON, $\quad(\operatorname{Sig}=0.046, \quad \mathrm{z}=-1 / 992)$.
Trichothecins are a large family of mycotoxins, the most toxic being T-2. The effect of this poison on the cause of Alzheimer's disease is very important. Different results from different studies have been reported on fungal toxins such as T-2 and DON. Yazdan Panah and his colleagues (2012), Lakzaie et al. (2014) tested 35 and 16 samples of wheat samples harvested in northern Iran (Gonbad, golestan) showing that all samples (100\%) were contaminated with T-2 toxin, and in a different study, showed that Fusarium toxins, including T-2 and DON, contaminated 24 types of human nutrition [10-12]. In this study, the effects of microorganisms induced by Argon plasma-induced microwave osmosis were studied against Fusarium mycotoxins such as DON and T2 toxins. Plasma treatment results showed a significant reduction in timedependent toxin concentrations. The plasma contains a gas mixture of charged particles (free electron and proton particles) and neutralized activated species, which include gas molecules, free radicals, and lowresolution ultraviolet photons. Highenergy electrons produce highly active chemical species, which is also due to collisions with atoms and molecules. In a gas mixture containing oxygen and water vapor, most of the primary radicals are $\mathrm{O}$ and $\mathrm{OH}$. When a reactor is launched at atmospheric pressure, a cool plasma jet is blown into the air. This plasma jet can also sterilize the surfaces. In hospitals, it can be used to eliminate bacteria and viruses and has the potential of replacing or using with autoclaves in the sterilization of surgical and dental instruments. In a study by Salama A Ouf and colleagues on the inhibitory effect of argon plasma refrigerated plasma on spores and mycotoxins produced by Aspergillus 
niger, they were able to significantly reduce spore and aspergillus toxins in test specimens of dried fruit under reaction with radicals $\mathrm{O}$ and $\mathrm{OH}$ and no trace of toxins of this fungus was observed in the test sample. In the same study, we performed the same results in the study of the effect of cold plasma decontamination on fusarium fungi and toxins of this fungus. In the samples, we also have no traces of fungi and toxins. In the face of the cool plasma, argon gas was not observed and the results were in perfect agreement with each other. These results indicate that the plasma system has a high potential not only to destroy mycotoxins, but also toxins produced from fungi, which can be greatly reduced and can be effectively applied in the food industry [13].

Therefore, with the advancement of the use of cold plasma for decontamination of food, it can be used to improve the effectiveness of this device in improving the quality of food in terms of contamination with microorganisms. Although physicochemical properties and structural changes in plasma-treated mycotoxins have not been defined, this study showed that the plasma system needed to eliminate and inactivate mycotoxins into specific conditions such as the time of action and the concentration of toxins in the food.

\section{Conclusion}

From the results of the present study, it can be concluded that the plasma system due to the high potential for detoxification is useful to involve in food industry.

\section{Acknowledgments}

The authors acknowledge special thanks to Deputy of Research and Technology, Islamic Azad University, Tehran, Iran.

\section{References}

[1] X. Lin, J.A. Alspaugh, H. Liu, S. Harris, Cold Spring. Medicine., 2014, 5, 654-679.

[2] W.G. Sorenson, Fungal spores, Environmental Health Perspectives., 1999, 107, 469-472.

[3] M. Muhammed, A. Theodora, D. Athanasios, K. Themistoklis, H. Kourkoumpetis, A. Carneiro, J. GlavisBloom, J. Coleman, Medicine., 2013, 92, 305-316.

[4] Y. Li, Z .Wang, R.C. Beier, J. Shen, D. De Smet, S. De Saeger, S. Zhang, J Agric Food Chem., 2011, 59, 3441-53; (b) N. Moradi kor, Asian Pac J Trop Med, 2014, 7, 282-288.

[5] G. Antonissen, A. Martel, F. Pasmans, R. Ducatelle, E. Verbrugghe, V. Vandenbroucke, L. Shaoji, F. Haesebrouck, F. Van Immerseel, S. Croubels, Toxins., 2014, 6, 430-452.

[6] J. Heinlin, G. Isbary, W. Stolz, G. Morfill, M. Landthaler, T. Shimizu, B. Steffes, T. Nosenko, J. Zimmermann, S. Karrer, $J$ Eur Acad Dermatol Venereol., 2011, 25, 1-11.

[7] R. Snoeckx, A. Bogaerts, Chem Soc Rev., 2017, 46, 5805-5863; (b) N. Moradi kor, Euro. J. Exp. Bio, 2014, 4, 340-348.

[8] M.G. Kong, G. Kroesen, G. Morfill, T. Nosenko, T. Shimizu, J. van, J.L. Zimmermann, New Journal of Physics., 2009, 11, 115- 150.

[9] L. Yang, J. Chen, J. Gao, Journal of Electrostatics., 2009, 67, 646-651; (B) N. Moradi kor, Int. J. Biosci, 2014, 4, 89-99.

[10] V. Dayaman, M. Kumar, M. Dua, A.K. dJohri, Frontiers in Microbiology., 2016, 7, 1083-1098.

[11] W.J. Rea, N. Didriksen, T. Simon, R. Pan, Y. Fenyves, E.J. Griffiths, Arch Environ Health., 2003, 58, 39940.

[12] H. Yazdanpanah, A. Zarghi, A.R. Shafaati, S.M. Foroutan, F. Aboul- 
Fathi, A. Khoddam, F. Nazari, IJPR., $\quad$ Abdelkawy, Journal of Physics., 2016, 2012, 11, 251-256. 49, 13079-13092.

[13] H.M. Abdel-Aleam, A. Eglal, A.

Hegazy Maha, W. Sayed Nour, M.

How to cite this manuscript: Elham Galin Abbasian, Mansour Bayat, Arash Chaichi Nosrati, Seyed Jamal Hashemi, Mahmood Ghoranneviss. The effect of atmospheric jet plasma on Fusarium species producing mycotoxins T2 and DON: An approach for physical and chemical investigation. Eurasian Chemical Communications, 2020, 2(3), 340-348. 